\title{
Noncentrosymmetric Commensurate Magnetic Ordering of Multiferroic $\operatorname{ErMn}_{2} \mathrm{O}_{5}$
}

\author{
B. Roessli ${ }^{1}$, P. Fischer ${ }^{1}$, P.J. Brown ${ }^{2}$, M. Janoschek ${ }^{1,3}$, D. \\ Sheptyakov $^{1}$, S.N. Gvasaliya ${ }^{1}$, B. Ouladdiaf ${ }^{3}$, O. Zaharko ${ }^{1}$, Eu. \\ Golovenchits $^{4}$, V. Sanina ${ }^{4}$ \\ ${ }^{1}$ Laboratory for Neutron Scattering, ETH Zurich \& Paul Scherrer Institut, CH-5232 \\ Villigen, PSI \\ ${ }^{2}$ Institut Laue-Langevin, 6 rue Jules Horowitz, BP 156, F-38042 Grenoble Cedex 9 \\ ${ }^{3}$ Technische Universität München, Physics Department E21, D-85748 Garching, \\ Germany \\ ${ }^{4}$ Ioffe Physical Thechnical Institute of Russian Academy of Science, Russia \\ E-mail: broessli@psi.ch
}

\begin{abstract}
The non-centrosymmetric magnetic structure of $\mathrm{ErMn}_{2} \mathrm{O}_{5}$ has been shown to be very similar to that of $\mathrm{HoMn}_{2} \mathrm{O}_{5}$ (Vecchini et al., 2008 Phys. Rev. B 77 134434). The magnetic modulation at $25 \mathrm{~K}$ has propagation vector $\vec{k}=(1 / 2,0,1 / 4)$ and the symmetry imposes very few constraints on the magnetic configurations allowed. Only by combining the results of bulk magnetisation measurements, powder and single crystal neutron diffraction and spherical neutron polarization analysis was it possible to distinguish clearly between different models. The susceptibility measurements show that the erbium magnetic moments are aligned parallel to the $c$-axis indicating strong single-ion anisotropy. Spherical neutron polarimetry demonstrates the presence of two unequally populated chirality domains in $\mathrm{ErMn}_{2} \mathrm{O}_{5}$ single crystals. X-ray diffraction measurements on an $\mathrm{ErMn}_{2} \mathrm{O}_{5}$ powder using synchrotron radiation show that the buckling angles of the Mn-O-Mn bond change below the transition to the ferroelectric phase.
\end{abstract}




\section{Introduction}

At the present time there is considerable interest in the often complex physics of multiferroic materials such as $\mathrm{RMn}_{2} \mathrm{O}_{5}$ oxides $(\mathrm{R}=$ rare earths, $\mathrm{Y}$, or $\mathrm{Bi})$, see for instance [1]-[3], and the papers cited therein. A systematic powder neutron diffraction study of $\mathrm{RMn}_{2} \mathrm{O}_{5}$ compounds at room temperature [4] confirmed the centrosymmetric space group Pbam (No. 55) for these compounds. The Mn ions occupy one set (Mn1) of $4 f(0,1 / 2, \mathrm{z})$ and one set $(\mathrm{Mn} 2) 4 h(\mathrm{x}, \mathrm{y}, 1 / 2)$ sites whilst the $\mathrm{R}$ atoms occupy $4 g(\mathrm{x}, \mathrm{y}, 0)$ positions. It was concluded from bond valence considerations and bulk magnetic measurements that the $4 f$ sites contain $\mathrm{Mn}^{4+}$ ions and the $4 h$ sites $\mathrm{Mn}^{3+}$ ions. For multiferroic ErMn $\mathrm{O}_{5}$ soft x-ray magnetic diffraction under an applied electric field confirms strong coupling between the ferroelectric- and antiferromagnetic order parameters [5].

Studies of magnetic ordering in $\mathrm{ErMn}_{2} \mathrm{O}_{5}$ date from 1973, when magnetic neutron diffraction peaks were observed at $20 \mathrm{~K}$ in a powder neutron diffraction investigation [6] and indexed with $\vec{k}=(1 / 2,0,0.242(5))$. Helical magnetic ordering of the Mn moments and sinusoidal modulation of the Er moments was proposed. An early single crystal neutron diffraction study of $\mathrm{ErMn}_{2} \mathrm{O}_{5}$ [7] concluded that the magnetic ordering of both Mn and Er was sinusoidal at $1.5 \mathrm{~K}$ and $25 \mathrm{~K}$ with $\vec{k}=(1 / 2,0,0.275(5))$ and $\mathrm{T}_{N}=44 \mathrm{~K}$.

The complex phase diagram of $\mathrm{ErMn}_{2} \mathrm{O}_{5}$ is described in ref. [8]. At all temperatures below the Néel temperature $\left(\mathrm{T}_{\mathrm{N}}=44 \mathrm{~K}\right)$ the magnetic structure is modulated with propagation vector $k$ close to $k_{x}=1 / 2, k_{y}=0, k_{z}=1 / 4$. Just below $\mathrm{T}_{\mathrm{N}}$ both $k_{x}$ and $k_{z}$ are incommensurate: the $2 \mathrm{D}-\mathrm{ICM}$ phase. On cooling below approximately $40 \mathrm{~K} k_{z}$ locks to $1 / 4$ giving the $1 \mathrm{D}-\mathrm{ICM}$ phase and finally at $37.7 \mathrm{~K} k_{x}$ locks in at $1 / 2$ and this commensurate (CM) phase is stable down to low temperature. Below about $10 \mathrm{~K}$, the CM phase coexists with a further $1 \mathrm{D}$-ICM phase with $k_{z}=0.271$ at $2 \mathrm{~K}$. Third order magnetic satellite peaks were observed in this phase indicating some squaring up of the sinusoidal modulation [9]. Below $\mathrm{T} \sim 35 \mathrm{~K}$ the compound is ferroelectric [10]. Further details of the phase diagram of $\mathrm{ErMn}_{2} \mathrm{O}_{5}$ have been elucidated by Fukunaga et al. [9] who made simultaneous measurements of magnetic neutron diffraction, electrical polarization and dielectric permittivity $\epsilon$. Weak ferroelectricity first appears in the 1D-ICM phase, but the peak of the permittivity occurs at the 1D-ICM to CM phase transition. Strangely the (1D-ICM) phase stable below $10 \mathrm{~K}$ is only weakly ferroelectric. This incommensurate magnetic low-temperature phase may be stabilized with respect to the commensurate phase by the application of external magnetic fields [10]. Hydrostatic pressure on the other hand has been shown to stabilize the commensurate ferroelectric phases of $\mathrm{RMn}_{2} \mathrm{O}_{5}(\mathrm{R}=\mathrm{Tb}, \mathrm{Dy}, \mathrm{Ho})$ [11.

The commensurate antiferromagnetic $(\mathrm{CM})$ structures of $\mathrm{RMn}_{2} \mathrm{O}_{5}(\mathrm{R}=\mathrm{Tb}$, Ho,

Dy; $\vec{k}=(1 / 2,0,1 / 4))$ have been determined by Blake et al. [12] from time-of-flight powder neutron diffraction data using group-theory symmetry arguments (concerning the latter see also ref. [2]). Unfortunately for this structure the crystal symmetry imposes rather few constraints on the variables to be determined. Nevertheless all 
the magnetic moments were found to lie in the (001) plane. An alternative structure for the commensurate phases of $\mathrm{RMn}_{2} \mathrm{O}_{5}(\mathrm{R}=\mathrm{Y}$, Ho, Er) has been proposed by Kimura et al. [13] based on single crystal neutron diffraction measurements. It is a rather complicated spiral chain model, described in terms of a $2 \vec{a} \times \vec{b} \times 4 \vec{c}$ magnetic super-cell, in which the magnetic moments have components along all three crystallographic axes. For ErMn $\mathrm{O}_{5}$ the major components of the Er magnetic moments were along the $c$-axis at $20 \mathrm{~K}$.

In the present paper we report measurements of the magnetic susceptibility of ErMn $_{2} \mathrm{O}_{5}$ single crystals as well as a combined unpolarized and polarized neutron scattering study of the magnetic structure of this compound at $\mathrm{T}=25 \mathrm{~K}$. We show that the magnetic ordering of $\mathrm{Mn}$ in ferroelectric $\mathrm{ErMn}_{2} \mathrm{O}_{5}$ is similar to that found in the Ho compound [14]. Moreover we demonstrate that for a structure containing several magnetic sublattices and few symmetry constraints, it is only by combining precise single crystal neutron diffraction intensity measurements with quantitative spherical neutron polarimetry [15] on the same specimens that different possible magnetic configurations may be clearly distinguished.

\section{Experimental techniques}

The single crystals of ErMn ${ }_{2} \mathrm{O}_{5}$ were grown by the flux method as described in [16]. Their magnetic response was studied by measuring both dynamic and static susceptibilities. The dynamic magnetic susceptibility in zero static magnetic field was measured by the induction method at a frequency of $10 \mathrm{kHz}$ in the temperature range $5-150 \mathrm{~K}$. The static magnetic susceptibility was obtained using a vibrating sample magnetometer in magnetic fields up to $30 \mathrm{kOe}$.

The neutron diffraction work was carried out partly at the Swiss spallation neutron source SINQ [17], Paul Scherrer Institut, Villigen, Switzerland (PSI) and partly at the high-flux reactor of the Institut Laue Langevin, Grenoble, France (ILL). Powder neutron diffraction measurements were made using the DMC diffractometer at PSI [18] and wavelength $\lambda=2.448 \AA$. The $\mathrm{ErMn}_{2} \mathrm{O}_{5}$ powder was contained in a cylindrical vanadium can $(6.5 \mathrm{~mm}$ diameter $50 \mathrm{~mm}$ high) under an atmosphere of He gas. The sample was maintained at stable temperatures down to $1.5 \mathrm{~K}$ in an ILL type ${ }^{4} \mathrm{He}$ flow cryostat. Single crystal diffraction measurements were made both at PSI and at ILL on two differently oriented samples of volume $\sim 50 \mathrm{~mm}^{3}$. The integrated intensities of reflections from the first crystal were measured on the four-circle single crystal neutron diffractometer TriCS at PSI [20] using a wavelength $\lambda=1.18 \AA$. For the second, the measurements were made with the four-circle diffractometer D10 at ILL using a wavelength $\lambda=1.526 \AA$. The neutron diffraction data were evaluated by means of a current version of the FullProf program package [21]. For the single crystal data neutron absorption was neglected, but an anisotropic extinction correction involving 6 parameters was made.

Because of the complexity of the magnetic structure of $\mathrm{ErMn}_{2} \mathrm{O}_{5}$, we have also 
made neutron polarimetric measurements on both crystals. For these measurements the polarimeter Cryopad 2 [15] was installed on the D3 diffractometer at ILL. An incident polarized beam wavelength $\lambda=0.82 \AA$ was obtained from a magnetised Heusler alloy monochromator and the polarization of the scattered beam analysed using a spin polarized ${ }^{3}$ He filter. The first specimen was oriented with an [010] axis vertical which gave access to reflections of type $h, 0, l$. With this orientation, neutron polarimetry measurements do not distinguish sensitively between the $a$ and the $c$-axis components of moment. We therefore made additional measurements on a second crystal oriented with a $[1,0, \overline{2}]$ axis vertical so that magnetic Bragg peaks with indices $h / 2, k, h / 4$ could be measured in the scattering plane.

\section{Bulk magnetic measurements}

The temperature dependence of the dynamic and static magnetic susceptibilities of $\mathrm{ErMn}_{2} \mathrm{O}_{5}$ along the three principal axes are shown in figures 1 and 2. The susceptibility measured along the $c$-axis is considerably higher than that in the two other axial directions. The susceptibilities along the $a$ - and $b$-axes show diffusive maxima at temperatures above $\mathrm{T}_{\mathrm{N}}$ and below $\mathrm{T} \sim 120 \mathrm{~K}$. Such behaviour is characteristic of shortrange magnetic correlations above $\mathrm{T}_{\mathrm{N}}$ that mask the anomaly in $\chi_{a, b}$. The susceptibility along the $c$-axis can be described by a Curie-Weiss law $\chi=C_{M} /\left(T-\theta_{p}\right)$ at all measured temperatures, although the slope of $\chi_{c}^{-1}(T)$ changes slightly near $\mathrm{T}=25 \mathrm{~K}$. No anomaly is observed around $\mathrm{T}_{\mathrm{N}}$, suggesting that the c-axis susceptibility is dominated by the magnetic response of Er. Fitting the susceptibility data to the Curie-Weiss law in the temperatures range $10-25 \mathrm{~K}$ gives $\theta_{p}=-5.6 \mathrm{~K}$ and an effective magnetic moment

$\mu \sim 7 \mu_{\mathrm{B}}$. This is rather less than the $\sim 9 \mu_{\mathrm{B}}$ expected for $\operatorname{Er}^{3+}\left({ }^{4} \mathrm{I}_{15 / 2}\right)$ with strong spin-orbit coupling.

The unit cell of $\mathrm{ErMn}_{2} \mathrm{O}_{5}$ contains three different magnetic species $\mathrm{Er}^{3+}, \mathrm{Mn}^{3+}$, and $\mathrm{Mn}^{4+}$. Since all crystals of the $\mathrm{RMn}_{2} \mathrm{O}_{5}$ family, including those with non magnetic $\mathrm{R}$ - ions ( $\mathrm{Y}$ and $\mathrm{Bi}$ ), have Néel temperatures $\mathrm{T}_{\mathrm{N}}$ near to $40 \mathrm{~K}$, it can be concluded that it is the manganese ions which order magnetically at $\mathrm{T}_{\mathrm{N}}$. Spontaneous antiferromagnetic ordering of Er occurs below $\left|\theta_{\mathrm{P}}\right| \sim 6 \mathrm{~K}$ and at higher temperatures the Er spins are polarized by the internal magnetic field arising from the Mn-Er exchange interaction. The susceptibility along the $c$-axis is paramagnetic at all temperatures up to the Néel temperature and is much higher than along the $a$ - and $b$-directions, showing that the $c$ axis is the easy direction of magnetization for the $\mathrm{Er}^{3+}$ moments. It is determined by the crystalline-electric-field anisotropy. On the other hand, the arrangement of the Er magnetic moments depends on the symmetry of the effective internal field which will be sensitive to spin reorientations in the Mn-sublattice. This is probably at the origin of the anomaly observed near $25 \mathrm{~K}$. 


\section{Magnetic structure of $\operatorname{ErMn}_{2} \mathrm{O}_{5}$ in the commensurate phase}

\subsection{Single crystal neutron diffraction}

In order to determine the magnetic structure of $\mathrm{ErMn}_{2} \mathrm{O}_{5}$ in the commensurate CM phase with propagation vector $\vec{k}=(1 / 2,0,1 / 4)$ two sets of integrated reflection intensities were collected on TriCS at SINQ from the first single crystal: 77 nuclear reflections were measured at $50 \mathrm{~K}$ in the paramagnetic state and 227 magnetic reflections at $30 \mathrm{~K}$. Subsequently 227 nuclear and 186 magnetic reflections were measured at $25 \mathrm{~K}$ from the second crystal on D10 at ILL. The nuclear intensities were used mainly to obtain the scale factor for the magnetic intensities. Using isotropic temperature factors

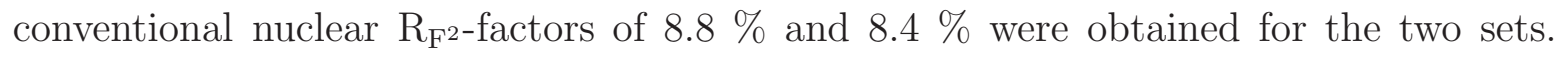
Within limits of error the refined structural parameters agree with the presumably more precise $20 \mathrm{~K}$ nuclear parameters of $\mathrm{ErMn}_{2} \mathrm{O}_{5}$ published in Ref. [13]. The positional parameters were then fixed to the latter values and an overall temperature factor $\mathrm{B}=$ 0 was used in the magnetic refinements.

The best fit of the magnetic reflections to a model in which the magnitudes of the Mn moments were kept constant was obtained with a magnetic Mn configuration similar to that found in $\mathrm{HoMn}_{2} \mathrm{O}_{5}$ [14]. This same configuration was also obtained using the simulated annealing procedure [21]. To conform to the easy [001] direction determined from the susceptibility measurements, the modulated magnetic Er moments were assumed to be oriented parallel to [001], in agreement with Gardner et al. [7]. This model gave magnetic $\mathrm{R}_{\mathrm{F}^{2}}$ factors of $12.1 \%\left(\mathrm{R}_{\mathrm{F}}=8.1 \%\right)$ and $15.6 \%\left(R_{F}=10.4 \%\right)$ for the $30 \mathrm{~K}$ and $25 \mathrm{~K}$ data sets, respectively. Using the helical configuration proposed in ref. [13] gave significantly worse magnetic fits: $\mathrm{R}_{\mathrm{F}^{2}}$ factors of $17.6 \%$ and $19.4 \%$ for 30 $\mathrm{K}$ and $25 \mathrm{~K}$, respectively.

Projections of the non-centrosymmetric commensurate magnetic structure proposed for $\mathrm{ErMn}_{2} \mathrm{O}_{5}$ at $30 \mathrm{~K}$ is shown in figure 4. The refined values of the magnetic parameters are summarized for $25 \mathrm{~K}$ and $30 \mathrm{~K}$ in Table 1 . Finally it is reassuring that the parameters of Table 1 yield, without further refinement, an excellent fit to the profile intensities measured at $\mathrm{T}=30 \mathrm{~K}$ on $\mathrm{DMC}$, with nuclear and magnetic $\mathrm{R}_{\text {Bragg }}$ factors of 1.9 and $10.5 \%$, respectively.

\subsection{Polarimetry}

The relationship between the incident and scattered polarizations $\vec{P}$ and $\vec{P}^{\prime}$ when a neutron beam is scattered by a magnetic system is given by the Blume-Maleev equations [22]. For magnetic Bragg scattering by an acentric antiferromagnetic structure

with finite propagation vector $\vec{k}$, such as the model proposed for $\operatorname{ErMn}_{2} \mathrm{O}_{5}$ in the previous section, these equations may be simplified to

$$
\begin{aligned}
\vec{P}^{\prime} I & =\vec{P}\left(-\left|\vec{M}_{\perp}\right|^{2}\right)+2 \Re\left[\vec{M}_{\perp}\left(\vec{P} \cdot \vec{M}_{\perp}^{*}\right)\right]-\Im\left(\vec{M}_{\perp} \times \vec{M}_{\perp}^{*}\right) \\
I & =\left|\vec{M}_{\perp}\right|^{2}+\vec{P} \cdot \Im\left(\vec{M}_{\perp} \times \vec{M}_{\perp}^{*}\right)
\end{aligned}
$$


where $\vec{M}_{\perp}$ is the magnetic interaction vector for the reflection. The quantities which were measured in the polarization analysis experiment are elements of the polarization matrix $P$ defined by

$$
P_{i}^{\prime}=P_{i j} P_{j}
$$

where $P_{i}$ and $P_{j}^{\prime}$ are components of the incident and scattered polarizations respectively defined on right-handed orthogonal cartesian polarization axes with $\vec{x}$ parallel to the scattering vector and $\vec{z}$ vertical. Using eqns 1 and 2 the polarization matrix for a single domain crystal can be written

$$
P\left|\vec{M}_{\perp}\right|^{2}=\left|\begin{array}{ccc}
-\left|\vec{M}_{\perp}\right|^{2} & 0 & 0 \\
-2 \Im\left(M_{\perp y} M^{*}{ }_{\perp z}\right) & \left|M_{\perp y}\right|^{2}-\left|M_{\perp z}\right|^{2} & 2 \Re\left(M_{\perp y} M^{*}{ }_{\perp z}\right) \\
-2 \Im\left(M_{\perp y} M^{*}{ }_{\perp z}\right) & 2 \Re\left(M_{\perp y} M^{*}{ }_{\perp z}\right) & \left|M_{\perp z}\right|^{2}-\left|M_{\perp y}\right|^{2}
\end{array}\right|
$$

The term $-2 \Im\left(M_{\perp y} M^{*}{ }_{\perp z}\right)$ is only present in chiral magnetic structures in which $\vec{M}_{\perp}$ and $\vec{M}_{\perp}^{*}$ are not parallel. It changes sign with the chirality of the structure. The term $2 \Re\left(M_{\perp y} M^{*}{ }_{\perp z}\right)$ may also have a different sign for different orientation domains. When more than one magnetic domain is present the measured elements are found by averaging over the domains with each weighted by its intensity $\left|\vec{M}_{\perp n}\right|^{2}$ and population $p_{n}$. In order to assess the number and the associated populations of the spin domains, all accessible symmetrically related Bragg reflections were measured. No evidence for the presence of orientation domains other than the two chirality domains was found. As a further check on the reproducibility of results, and possible aberrations of the polarimeter, the measurements were made with both positive and negative incident polarizations. The scattered polarizations were corrected for the analyser efficiency which was determined by measuring the polarization scattered by a nuclear reflection at intervals throughout the experiment. The independent elements of the polarization matrix which can be measured for this type of magnetic structure are:

$$
\begin{array}{ll}
P_{y x}=-2 \eta_{c} \Im\left(M_{\perp y} M^{*}{ }_{\perp z}\right) /\left|\vec{M}_{\perp}\right|^{2}, & \eta_{c}=\frac{p_{+}-p_{-}}{p_{+}+p_{-}} \\
P_{y y}=\left(\left|M_{\perp y}\right|^{2}-\left|M_{\perp z}\right|^{2}\right) /\left|\vec{M}_{\perp}\right|^{2}, & P_{y z}=2 \Re\left(M_{\perp y} M^{*}{ }_{\perp z}\right) /\left|\vec{M}_{\perp}\right|^{2}
\end{array}
$$

where $p_{+}$and $p_{-}$are the populations of the two chirality domains

Table 2 shows the independent elements of the polarization matrices obtained in the CM phase at $25 \mathrm{~K}$, for the first and second crystal orientations, respectively. They have been averaged using the relationships between the elements of the polarization matrices:

$$
\begin{array}{rlrl}
P_{x x}= & -P_{-x x}=-1 \\
P_{x y}= & P_{x z}=P_{-x y}=P_{-x z}=0 \\
P_{y x}=P_{z x}=P_{-y x}=P_{-z x} \\
P_{y y}=-P_{z z}=-P_{-y y}=P_{-z z} \\
P_{y z}=P_{z y}=-P_{-y z}=-P_{-z y}
\end{array}
$$

and between elements of matrices of symmetrically related reflections:

$$
\begin{aligned}
& P_{i j}(h k l)=P_{i j}(\bar{h} k \bar{l})=P_{i j}(h \bar{k} l)=P_{i j}(\bar{h} \bar{k} \bar{l}) \quad \text { for } \quad i j=y x, y y \\
& P_{i j}(h k l)=P_{i j}(\bar{h} k \bar{l})=-P_{i j}(h \bar{k} l)=-P_{i j}(\bar{h} \bar{k} \bar{l}) \text { for } \quad i j=y z
\end{aligned}
$$


The standard deviations, given in parentheses, have been estimated from the deviations of individual measurements from the means.

Neither of the two magnetic structures which had previously been proposed for $\mathrm{ErMn}_{2} \mathrm{O}_{5}$ are compatible with the polarimetric measurements. In the structure suggested by Buisson [6] the Mn moments on each sub-lattice rotate in the $a-b$ plane with a helical modulation propagating in the $c$ direction, the phase relationships between the different Mn sub-lattices lead to a canted arrangement of moments in the Mn planes at $z=0, z \approx \pm \frac{1}{4}$ and $z=\frac{1}{2}$. Although the chiral character of this structure can account for the finite values observed for $P_{y x}$, the $P_{y y}$ elements calculated for the $\frac{3}{2} 0 \frac{3}{4}$ and $\frac{5}{2} 0 \frac{7}{4}$ reflections are very small: 0.055 and -0.020 respectively whereas the observed values: $0.90(2)$ and $0.57(5)$ are much larger. On the other hand in the magnetic structure proposed for $\mathrm{ErMn}_{2} \mathrm{O}_{5}$ by Gardner et al. [7] the Er and Mn-spins are confined to the $a-c$ plane and hence the magnetic interaction vectors can have no component parallel to the $\mathrm{b}^{*}$-axis. In this case the chiral part of the neutron cross-section $\left(\vec{M}_{\perp} \times \vec{M}_{\perp}^{*}\right)$ vanishes for the $h 0 l$ reflections so that this model cannot account for the finite $P_{y x}$ observed. It can be concluded that the magnetic structure of $\mathrm{ErMn}_{2} \mathrm{O}_{5}$ must contain spin components along the b-axis. The helical model of the magnetic structure of $\mathrm{ErMn}_{2} \mathrm{O}_{5}$ recently proposed by Kimura [13] fulfills this criterion: the Mn-spins have a spiral modulation with components in both the $(a, c)$ and $(b, c)$-planes and the Ermoments are parallel to the $c$-axis and have a sine-wave modulation. The elements of the polarization matrix calculated with this model yield much better agreement with the observations than either of the two structures discussed previously. For the $\frac{3}{2} 0 \frac{3}{4}$ Bragg reflection:

$$
\begin{array}{lllc}
P_{y x}=-0.40 & P_{y y}=0.72 & P_{y z}=0 & \text { calculated ref. [13] } \\
P_{y x}=-0.32 & P_{y y}=0.87 & P_{y z}=0.06 & \text { observed }
\end{array}
$$

This model of the magnetic structure of $\mathrm{ErMn}_{2} \mathrm{O}_{5}$ at $\mathrm{T}=25 \mathrm{~K}$ gives qualitative agreement with the polarization data. However, a least-square fit to the complete set of polarimetric data taking into account the two unequally populated chirality domains yielded, at best, $\chi^{2}=68$. This suggests, in agreement with the single crystal diffraction data, that Kimura's model is not adequate.

The magnetic model which gave the best fit to the integrated intensity data is that shown in Table 1 which is similar to the magnetic structure of $\mathrm{HoMn}_{2} \mathrm{O}_{5}$ [14 in the commensurate phase. The results obtained by fitting this model to the polarimetric data, is shown in Table 1 . The starting parameters were those obtained from the single crystal integrated intensity measurements and because the absolute size of the magnetic moments cannot be determined from neutron polarimetry when the propagation vector is non-zero, the $x$-component of the magnetic moment of $\mathrm{Mn}^{3+}$ was fixed. The fit obtained, indicated by $\chi^{2}=8$, is very much better that obtained for any of the other structures tried. For this model, the only orientation domains are those related by the inversion symmetry. These two domains have spin structures with opposite chiralities. 
The polarimetric measurements allow the chiral domain fraction $\eta_{c}$ to be determined. The inequality in population of the chiral domains in the two crystals studied were found to be very similar: the values determined were $\eta_{c}=0.74(2)$ and $0.70(2)$ for the $[010]$ and $[10 \overline{2}]$ crystals respectively.

\section{X-ray diffraction with synchrotron radiation}

A synchrotron X-ray diffraction measurement on a powder sample of $\operatorname{ErMn}_{2} \mathrm{O}_{5}$ was made to search for any lattice distortion which may occur on passing into the ferroelectric phase. The powder sample was contained in a glass capillary with a diameter of 0.3 $\mathrm{mm}$. Diffraction patterns were collected at the high resolution powder diffraction station of the Materials Sciences beam-line at the Swiss Light Source (SLS). The wavelength was $\lambda=0.618 \AA$. All the data were taken whilst heating between $5 \mathrm{~K}$ and $71 \mathrm{~K}$ in steps of $2 \mathrm{~K}$. The diffraction lines observed as well as those found to be systematically absent were consistent with either of the space groups Pbam or Pba2. In fact the data are equally consistent with $P b 2_{1} m$ since the additional reflections allowed by $P b 2_{1} m$; $(h 0 l$ with $h$ odd) have zero intensity within the accuracy of the measurements. In addition no evidence for superlattice reflections corresponding to a lattice distortion of twice the magnetic propagation vector could be observed in our measurements on contrary to recent single crystal synchrotron investigations in $\mathrm{HoMn}_{2} \mathrm{O}_{5}$ [23]. The data therefore cannot confirm whether the true space group of the low-temperature crystal structure is lower than Pbam. The quality of the refinements was practically identical using any of the three space groups. The agreement factors are slightly better for $P b 2_{1} m$, perhaps due to the higher number of parameters refined. We have therefore chosen to discuss the results obtained for the refinement of the data in space group Pbam in which there are the fewest parameters to be refined. This yielded excellent fits at all temperatures with space group Pbam and isotropic temperature factors, e.g. at $25 \mathrm{~K}$ the agreement values: $\mathrm{R}_{\text {Bragg }}=4.4 \%, \mathrm{R}_{\mathrm{wp}}=11.2 \%$ and goodness of fit $\chi^{2}=3.1$. Figure 5 shows that there are clear changes in the crystal structure, as reflected by changes in the unit cell parameters, below the temperature of the ferroelectric transition. A study of the evolution of the structural parameters through the ferroelectric phase transition shows that it is the square pyramids of oxygen coordinating the $\mathrm{Mn}^{3+}$ ions that are most affected, see fig. 6. The oxygen atoms located in the base plane of the pyramids move along the $c$-axis at $\mathrm{T} \sim \mathrm{T}_{C}$ so that the $\mathrm{Mn}^{4+}{ }_{-} \mathrm{O}-\mathrm{Mn}^{3+}$ and $\mathrm{Mn}^{3+}{ }_{-} \mathrm{O}-\mathrm{Mn}^{3+}$ bond angles decrease on cooling. A sketch of the buckling distortion is shown in fig. 7. The $\mathrm{Mn}^{3+}-\mathrm{Mn}^{3+}$ and $\mathrm{Mn}^{3+}-\mathrm{Mn}^{4+}$ distances however remain constant.

\section{Discussion}

As pointed out in the introduction the zero-field magnetic phase diagram of $\mathrm{ErMn}_{2} \mathrm{O}_{5}$ is complex and the crystal undergoes a series of magnetic phase transitions 2D$\mathrm{CM} 1 \Rightarrow 1 \mathrm{D}-\mathrm{ICM} \Rightarrow \mathrm{CM} \Rightarrow 1 \mathrm{D}-\mathrm{ICM}$ on cooling below $\mathrm{T}_{\mathrm{N}}$. The exchange interactions in 
$\mathrm{RMn}_{2} \mathrm{O}_{5}$ ( $\mathrm{R}=$ Rare-earth, $\mathrm{Bi}$ ) have not yet been determined with precision but this series of ICM-CM-ICM transitions is characteristic of frustrated and competing exchange interactions [25]. In addition, centro-symmetry is lost in all the ordered magnetic phases allowing to ferroelectricity and leading to a Dzyaloshinskii-Moriya interaction. Combination of these two effects is probably the cause of both the non-collinearity and the small cycloidal component in the magnetic structure of the commensurate phase.

The magnetic structure of $\mathrm{ErMn}_{2} \mathrm{O}_{5}$ in the commensurate phase is very similar to the one found for the commensurate phase of $\mathrm{HoMn}_{2} \mathrm{O}_{5}$ [14 although in the Er compound the stronger single-ion anisotropy forces the Er-spins to be aligned along the $c$-axis. Due to the Er-Mn exchange field, the Mn magnetic moments also have a larger $c$-component than found in the Ho compound.

Ferroelectric polarization with $\vec{k}=0$, aligned along the $b$-axis, coexisting with magnetic ordering with non-zero $\vec{k}$, is allowed by the symmetry of Pbam on all the Mn and Er sublattices for the irreproducible representation $\Gamma_{4}$. Similar symmetry analyses have been made in Ref. 24] and Ref. [3] for $\mathrm{RMn}_{2} \mathrm{O}_{5}$ crystals. Actually the point group symmetry of the magnetic structure determined here is monoclinic, retaining only the mirror plane perpendicular to $\vec{a}$. Already the fact that the mirror plane perpendicular to the $c$ axis is missing in the group $\mathrm{G}_{\vec{k}}$ of the propagation vector $(m m 2)$ implies that the magnetic structure is non-centrosymmetric, and the $\mathrm{Mn}^{4+}$ ions can split into two orbits. In addition the 2-fold rotation around the $c$-axis does not leave the magnetic configuration invariant. The symmetry of the magnetic structure is therefore only $P b^{\prime}$.

\section{Conclusion}

In conclusion, we have determined the magnetic structure of $\mathrm{ErMn}_{2} \mathrm{O}_{5}$ in the commensurate phase. The arrangement of the Mn moments is very similar to that determined for $\mathrm{HoMn}_{2} \mathrm{O}_{5}$ [14. This structure is in good agreement with powder, single crystal diffraction and neutron polarimetry measurements. It may be noted that the alternative structure proposed for $\mathrm{ErMn}_{2} \mathrm{O}_{5}$ [13] gives only slightly worse agreement with the integrated intensity data but can clearly be rejected using the polarimetric results. Because of the strong crystal field anisotropy the magnetic moments of Er are aligned along the $c$-axis and induce a larger $c$-component of the Mn-spins than that found in $\mathrm{HoMn}_{2} \mathrm{O}_{5}$. Neutron polarimetry reveals that the ferroelectric phase is characterised by unequal population of the two chirality domains. The X-ray diffraction measurements show that the average crystallographic structure distorts in the ferroelectric phase transition. The principal effect of the distortion is to change the buckling angles of the Mn-O-Mn bonds leaving the distances between Mn-ions unchanged.

\section{Acknowledgments}

The authors are grateful to the ILL for allocating beam-time for this experiment. Part of this work was done at the Paul Scherrer Institut, Switzerland. B.R. would like to 
thank L.C. Chapon and H. Grimmer for useful discussions. This work was partially supported by RFBR (Nr. 08-02-00077) and Presidium of RAS (Nr. 03).

\section{References}

[1] Harris A B 2007 Phys. Rev. B 76 054447; Harris A B, Kenzelmann M, Aharony A, Entin-Wohlman O, 2008 Phys. Rev. B 78014407

[2] Chapon L C, Radaelli P G, Blake G R, Park S, Cheong S-W 2006 Phys. Rev. Lett. 96097601

[3] Kadomtseva A M, Krotov S S, Popov Yu F, Vorob'ev G P, Lukina M M 2005 JETP 100305

[4] Alonso J A, Casai M T, Martinez-Lope M J, Martinez J L, Fernandez-Diaz M T 1997 J. Phys.: Condens. Matter 98515

[5] Bodenthin Y, Staub U, Garcia-Fernandez M, Janoschek M, Schlappa J, Golovenchits E I, Sanina V A, Lushnikov S G 2008 Phys. Rev. Lett. 100027201

[6] Buisson G 1973 phys. stat.sol. (a) 16 533; Buisson G 1973 phys. stat.sol. (a) 17191

[7] Gardner P P, Wilkinson C, Forsyth J B, Wanklyn B M 1988 J. Phys. C: Solid State Phys. 215653

[8] Kobayashi S, Osawa T, Kimura H, Noda Y, Kagomiya I, Kohn K 2004 J. Phys. Soc. Japan 73 1031

[9] Fukunaga M, Nishihata K, Kimura H, Noda Y, Kohn K 2007 J. Phys, Soc. Japan 76074710

[10] Higashiyama D, Miyasaki S, Tokura Y 2005 Phys. Rev. B 72064421

[11] de la Cruz C R, Lorenz B, Sun Y Y, Wang Y, Park S, Cheong S-W, Gospodinov M M, Chu C W 2007 Phys. Rev. B 76 174106; Kimura H, Nishihata K, Noda Y, Aso N, Matsubayashi K, Uwatoko Y, Fujiwara T, 2008 J. Phys. Soc. Japan 774

[12] Blake G R, Chapon L C, Radaelli P G, Park S, Hur N, Cheong S-W, Rodriguez-Carvajal J 2005 Phys. Rev. B 71214402

[13] Kimura H, Fukuda S K Y, Osawa T, Kamada Y N Y, Kagomiya I, Kohn K, 2007 J. Phys, Soc. Japan $\mathbf{7 6} 074706$

[14] Vecchini C, Chapon L C, Brown P J, Chatterji T, Park S, Cheong S-W, Radaelli P G 2008 Phys. Rev. B 77134434

[15] Tasset F, Brown P J, Lelivre-Berna E, Roberts T, Pujol S, Allibon J, Bourgeat-Lami E 1999 Physica B 267-268 69

[16] Sanina V A, Sapozhnikova L M, Golovenchits E I, Morozov N V, 1988 Sov. Phys. Sol. St., 30 1736; Golovenchits E I, Morozov N V, Sanina V A, Sapozhnikova L M, 1992 Sov. Phys. Sol. St. 3456 (199)

[17] Fischer W E 1997 Physica B 234-236 1202

[18] Fischer P, Keller L, Schefer J, Kohlbrecher J 2000 Neutron News 1119

[19] Fischer P, Frey G, Koch M, Knnecke M, Pomjakushin V, Schefer J, Thut R, Schlumpf N, Brge R, Greuter U, Bondt S, Berruyer E 2000 Physica B 276-278 168

[20] Schefer J, Könnecke M, Murasik A, Czopnik A, Strässle Th, Keller P, Schlumpf N 2000 Physica B 276-278 168

[21] Rodriguez-Carvajal J 1993 Physica B 19255

[22] Blume M 1963 Phys. Rev. 130 1670; Maleev S V, Baryaktar V G, Suris R A,(1963) Sov. Phys. Solid State 42533

[23] Beutier G, Bombardi A, Vecchini C, Radaelli P.G., Park S, Cheong S.-W., Chapon L.C. 2008 Phys. Rev. B 77, 172408

[24] Kagomiya I, Matsumoto S, Kohn K, Fukuda Y, Shoubu T, Kimura H, Noda Y, Ikeda N, 2003 Ferroelectrics 286167

[25] Yu. A Izyumov, 1984 Sov. Phys. Usp. 27845 
Table 1: Magnetic parameters of $\operatorname{ErMn}_{2} \mathrm{O}_{5} . \quad M$ and $I$ are the real and imaginary magnetic Fourier components and magnitudes in units of $\mu_{B}$ and phase in units of $2 \pi$, refined from single crystal neutron intensities at $30 \mathrm{~K}$ and $25 \mathrm{~K}$ (upper and lower values, respectively). The third row is the result of the calculations of the magnetic Fourier components from the polarimetry data taken at $\mathrm{T}=25 \mathrm{~K}$. Estimated standard deviations of the parameters are given within brackets and refer to the last relevant digit.

\begin{tabular}{|c|c|c|c|c|c|c|c|c|c|c|c|c|}
\hline Ion & $\mathrm{x}$ & $\mathrm{y}$ & $\mathrm{z}$ & $M_{x}$ & $M_{y}$ & $M_{z}$ & M & $I_{x}$ & $I_{y}$ & $I_{z}$ & I & phase \\
\hline \multirow[t]{3}{*}{ Er1 } & 0.137 & 0.171 & 0 & 0 & 0 & $0.3(1)$ & $0.3(1)$ & 0 & 0 & 0 & 0 & 0.250 \\
\hline & & & & 0 & 0 & $0.5(1)$ & $0.5(1)$ & 0 & 0 & 0 & 0 & 0.250 \\
\hline & & & & 0 & 0 & $0.65(1)$ & $0.65(1)$ & 0 & 0 & 0 & 0 & 0.250 \\
\hline \multirow[t]{3}{*}{ Er2 } & 0.863 & 0.829 & 0 & 0 & 0 & $1.6(1)$ & $1.6(1)$ & 0 & 0 & 0 & 0 & 0.250 \\
\hline & & & & 0 & 0 & $1.7(2)$ & $1.7(2)$ & 0 & 0 & 0 & 0 & 0.250 \\
\hline & & & & 0 & 0 & $1.80(5)$ & $1.80(5)$ & 0 & 0 & 0 & 0 & 0.250 \\
\hline \multirow[t]{3}{*}{ Er3 } & 0.637 & 0.329 & 0 & 0 & 0 & $-1.4(1)$ & $1.4(1)$ & 0 & 0 & 0 & 0 & 0.250 \\
\hline & & & & 0 & 0 & $-1.7(2)$ & $1.7(2)$ & 0 & 0 & 0 & 0 & 0.250 \\
\hline & & & & 0 & 0 & $-1.80(5)$ & $1.80(5)$ & 0 & 0 & 0 & 0 & 0.250 \\
\hline \multirow[t]{3}{*}{ Er4 } & 0.363 & 0.671 & 0 & 0 & 0 & $0.1(1)$ & $0.1(1)$ & 0 & 0 & 0 & 0 & 0.250 \\
\hline & & & & 0 & 0 & $0.4(1)$ & $0.4(1)$ & 0 & 0 & 0 & 0 & 0.250 \\
\hline & & & & 0 & 0 & $0.65(1)$ & $0.65(1)$ & 0 & 0 & 0 & 0 & 0.250 \\
\hline \multirow[t]{3}{*}{$\mathrm{Mn}^{3+} 1$} & 0.412 & 0.350 & 0.5 & $3.28(5)$ & $-0.82(9)$ & 0 & $3.38(5)$ & 0 & 0 & $0.82(6)$ & $0.82(6)$ & 0.125 \\
\hline & & & & $3.34(7)$ & $-1.0(1)$ & & $3.50(6)$ & 0 & 0 & $0.85(8)$ & $0.85(8)$ & 0.125 \\
\hline & & & & 3.37 & $-0.98(2)$ & & 3.51 & 0 & 0 & $1.23(4)$ & $1.23(4)$ & 0.125 \\
\hline \multirow[t]{3}{*}{$\mathrm{Mn}^{3+} 2$} & 0.588 & 0.650 & 0.5 & $-3.28(5)$ & $0.82(9)$ & 0 & $3.38(5)$ & 0 & 0 & $-0.82(6)$ & $0.82(6)$ & 0.125 \\
\hline & & & & $-3.34(7)$ & $1.0(1)$ & 0 & $3.50(6)$ & 0 & 0 & $-0.85(8)$ & $0.85(8)$ & 0.125 \\
\hline & & & & -3.37 & $0.98(2)$ & 0 & 3.51 & 0 & 0 & $-1.23(4)$ & $1.23(4)$ & 0.125 \\
\hline \multirow[t]{3}{*}{$\mathrm{Mn}^{3+} 3$} & 0.912 & 0.150 & 0.5 & $-3.28(5)$ & $-0.82(9)$ & 0 & $3.38(5)$ & 0 & 0 & $0.82(6)$ & $0.82(6)$ & 0.125 \\
\hline & & & & $-3.34(7)$ & $-1.0(1)$ & 0 & $3.50(6)$ & 0 & 0 & $0.85(8)$ & $0.85(8)$ & 0.125 \\
\hline & & & & -3.37 & $-0.98(2)$ & 0 & 3.51 & 0 & 0 & $1.23(4)$ & $1.23(4)$ & 0.125 \\
\hline \multirow[t]{3}{*}{$\mathrm{Mn}^{3+} 4$} & 0.088 & 0.850 & 0.5 & $-3.28(5)$ & $-0.82(5)$ & 0 & $3.38(5)$ & 0 & 0 & $0.82(6)$ & $0.82(6)$ & 0.125 \\
\hline & & & & $-3.34(5)$ & $-1.0(1)$ & 0 & $3.50(6)$ & 0 & 0 & $0.85(8)$ & $0.85(8)$ & 0.125 \\
\hline & & & & -3.37 & $-0.98(2)$ & 0 & 3.51 & 0 & 0 & $1.23(4)$ & $1.23(40$ & 0.125 \\
\hline \multirow[t]{3}{*}{$\mathrm{Mn}^{4+} \mathrm{a} 1$} & 0 & 0.5 & 0.254 & $-2.09(5)$ & $0.71(9)$ & 0 & $2.20(5)$ & 0 & 0 & $-0.92(5)$ & $0.92(5)$ & $0.083(3)$ \\
\hline & & & & $-2.27(7)$ & $0.4(1)$ & 0 & $2.3(6)$ & 0 & 0 & $-1.08(7)$ & $1.08(7)$ & $0.081(4)$ \\
\hline & & & & $-2.36(7)$ & $0.39(1)$ & 0 & $2.39(7)$ & 0 & 0 & $-1.08(3)$ & $1.08(3)$ & $0.085(5)$ \\
\hline \multirow[t]{3}{*}{$\mathrm{Mn}^{4+} \mathrm{a} 2$} & 0.5 & 0 & 0.254 & $2.09(5)$ & $0.71(9)$ & 0 & $2.20(5)$ & 0 & 0 & $-0.92(5)$ & $0.92(5)$ & $0.083(3)$ \\
\hline & & & & $2.27(7)$ & $0.4(1)$ & 0 & $2.31(6)$ & 0 & 0 & $-0.82(7)$ & $0.82(7)$ & $0.081(4)$ \\
\hline & & & & $2.36(7)$ & $0.39(1)$ & 0 & $2.39(7)$ & 0 & 0 & $-1.08(3)$ & $1.08(3)$ & $0.085(5)$ \\
\hline \multirow[t]{3}{*}{$\mathrm{Mn}^{4+} \mathrm{b} 1$} & 0.5 & 0 & 0.746 & $2.09(5)$ & $0.71(9)$ & 0 & $2.20(5)$ & 0 & 0 & $-0.92(5)$ & $0.92(5)$ & $0.167(3)$ \\
\hline & & & & $2.27(7)$ & $0.4(1)$ & 0 & $2.31(6)$ & 0 & 0 & $-0.82(7)$ & $0.82(7)$ & $0.169(4)$ \\
\hline & & & & $2.36(7)$ & $0.39(1)$ & 0 & $2.39(7)$ & 0 & 0 & $-1.08(3)$ & $1.08(3)$ & $0.165(5)$ \\
\hline \multirow[t]{3}{*}{$\mathrm{Mn}^{4+} \mathrm{b} 2$} & 0 & 0.5 & 0.746 & $-2.09(5)$ & $0.71(9)$ & 0 & $2.20(5)$ & 0 & 0 & $-0.92(5)$ & $0.92(5)$ & $0.167(3)$ \\
\hline & & & & $-2.27(7)$ & $0.4(1)$ & 0 & $2.31(6)$ & 0 & 0 & $-0.82(7)$ & $0.82(7)$ & $0.169(4)$ \\
\hline & & & & $-2.36(7)$ & $0.39(1)$ & 0 & $2.39(7)$ & 0 & 0 & $-1.08(3)$ & $1.08(3)$ & $0.165(5)$ \\
\hline
\end{tabular}


Table 2: Observed and calculated values of independent elements of the polarization matrices for $\mathrm{ErMn}_{2} \mathrm{O}_{5}$ at $\mathrm{T}=25 \mathrm{~K}$ obtained with Cryopad on D3.

\begin{tabular}{rcccrrrrrr}
\hline \multirow{2}{*}{-axis } & $h$ & $k$ & $l$ & \multicolumn{2}{c}{$P_{y x}$} & \multicolumn{2}{c}{$P_{y y}$} & \multicolumn{2}{c}{$P_{y z}$} \\
& & & & \multicolumn{1}{c}{ Obs } & Calc & \multicolumn{1}{c}{ Obs } & Calc & \multicolumn{1}{c}{ Obs } & Calc \\
\hline \multirow{6}{*}[010]{} & 1.50 & 0.00 & 0.25 & $0.54(3)$ & 0.53 & $-0.71(3)$ & -0.69 & $-0.06(3)$ & 0.01 \\
& 1.50 & 0.00 & 0.75 & $0.35(2)$ & 0.33 & $0.90(2)$ & 0.90 & $0.06(2)$ & 0.00 \\
& 0.50 & 0.00 & 1.75 & $-0.40(4)$ & -0.35 & $0.85(2)$ & 0.88 & $0.09(3)$ & 0.00 \\
& 2.50 & 0.00 & 1.75 & $-0.62(4)$ & -0.54 & $0.57(5)$ & 0.69 & $0.11(4)$ & 0.00 \\
& 0.50 & 0.00 & 2.25 & $-0.32(2)$ & -0.29 & $0.89(1)$ & 0.92 & $0.07(2)$ & 0.00 \\
\hline \multirow{6}{*}[10\overline{2}]{} & 0.50 & 0.00 & 0.25 & $0.49(5)$ & 0.12 & $-0.68(9)$ & -0.99 & $0.04(6)$ & 0.00 \\
& 2.50 & 0.00 & 1.25 & $0.23(4)$ & 0.31 & $-0.95(2)$ & -0.88 & $0.04(3)$ & 0.00 \\
& 0.50 & 1.00 & 0.25 & $-0.50(2)$ & -0.48 & $-0.62(1)$ & -0.63 & $-0.31(1)$ & -0.30 \\
& 0.50 & 2.00 & 0.25 & $-0.61(2)$ & -0.62 & $0.33(1)$ & 0.33 & $-0.35(1)$ & -0.34 \\
& 1.50 & 2.00 & 0.75 & $0.62(2)$ & 0.63 & $-0.32(1)$ & -0.25 & $-0.29(1)$ & -0.36 \\
& 0.50 & 3.00 & 0.25 & $-0.16(1)$ & -0.14 & $0.39(1)$ & 0.37 & $-0.89(1)$ & -0.91 \\
& 1.50 & 3.00 & 0.75 & $0.26(2)$ & 0.36 & $0.61(2)$ & 0.70 & $0.69(3)$ & 0.50 \\
\hline
\end{tabular}

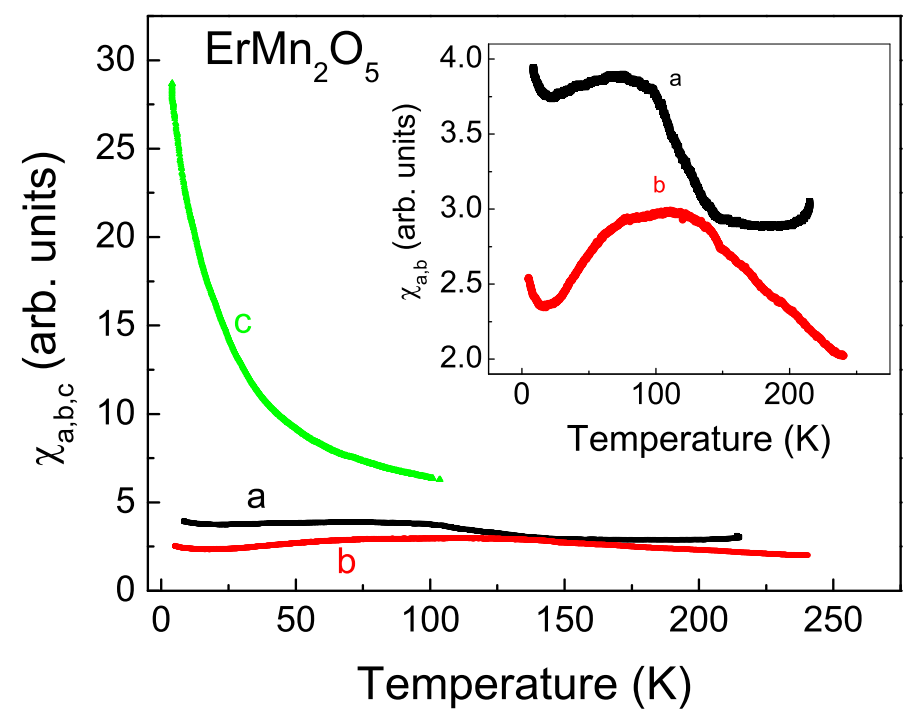

Figure 1: The temperature dependence of the dynamic magnetic susceptibilities along the main axes of $\mathrm{ErMn}_{2} \mathrm{O}_{5}$. 


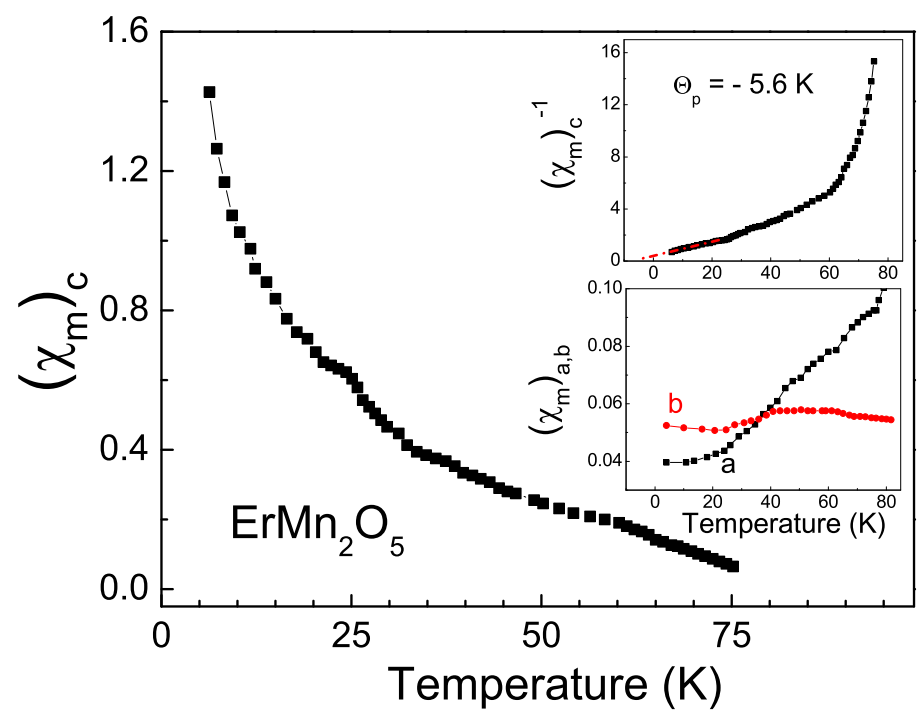

Figure 2: The temperature dependence of the molar magnetic susceptibility of $\mathrm{ErMn}_{2} \mathrm{O}_{5}$ along the $c$-axis. Upper insert: The same dependence for the inverse molar susceptibility along the $c$-axis. Lower inset: Temperature dependencies of the molar magnetic susceptibility along the $a$ and $b$-axes.
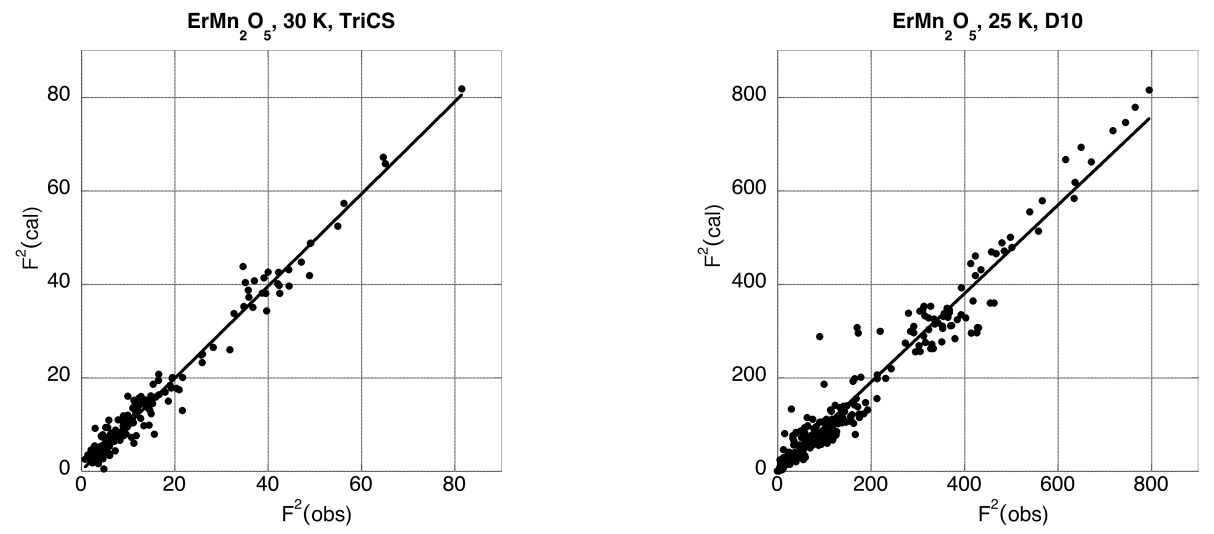

Figure 3: Calculated magnetic neutron intensities $\left(\mathrm{F}^{2}\right)$ versus observed values for $\mathrm{ErMn}_{2} \mathrm{O}_{5}$ at a) $30 \mathrm{~K}$ and b) at $25 \mathrm{~K}$, respectively. 


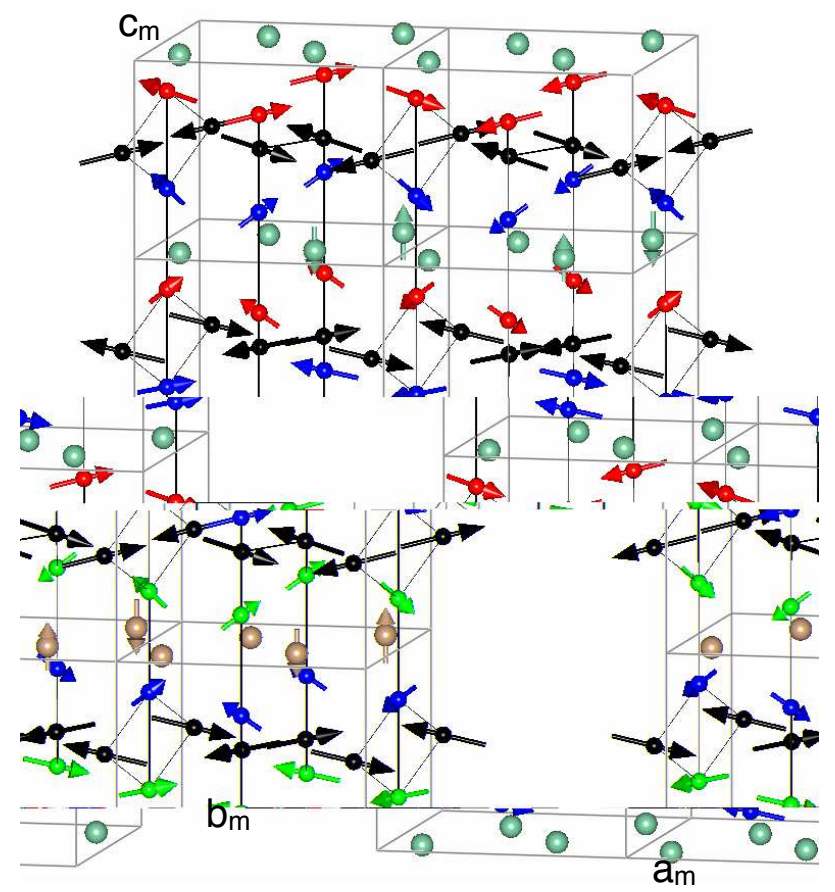

Figure 4: Magnetic unit cell of $\mathrm{ErMn}_{2} \mathrm{O}_{5}$ at $30 \mathrm{~K}$, plotted by means of program Studio [21]. $\mathrm{Mn}^{3+}$ ions are shown in black, $\mathrm{Er}^{3+}$ ions in green and the two orbits of $\mathrm{Mn}^{4+}$ in blue and red, respectively. Note the noncentrosymmetry of the magnetic structure, in particular due to these different orbits. 
Noncentrosymmetric Commensurate Magnetic Ordering of Multiferroic $\mathrm{ErMn}_{2} \mathrm{O}_{5}$
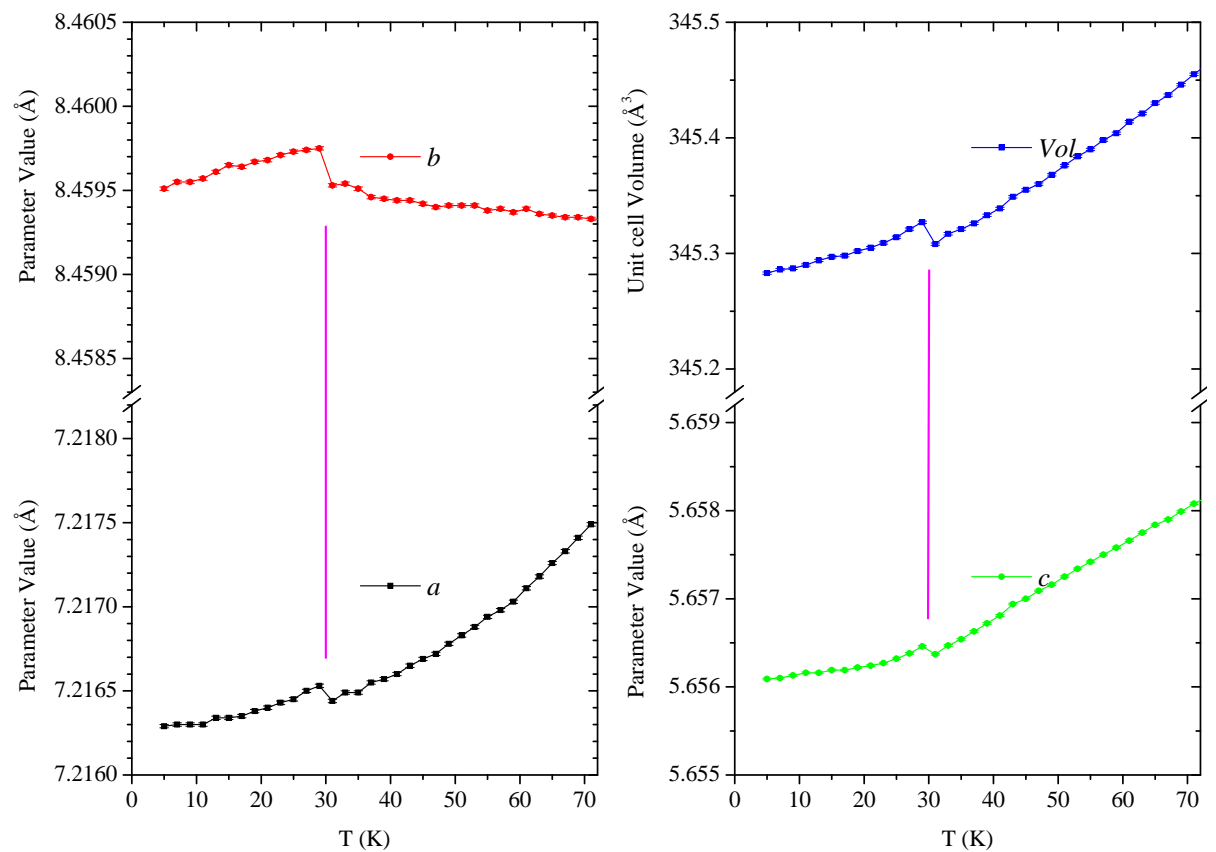

Figure 5: Temperature evolution of the lattice parameters of $\mathrm{ErMn}_{2} \mathrm{O}_{5}$ through the ferroelectric transition.

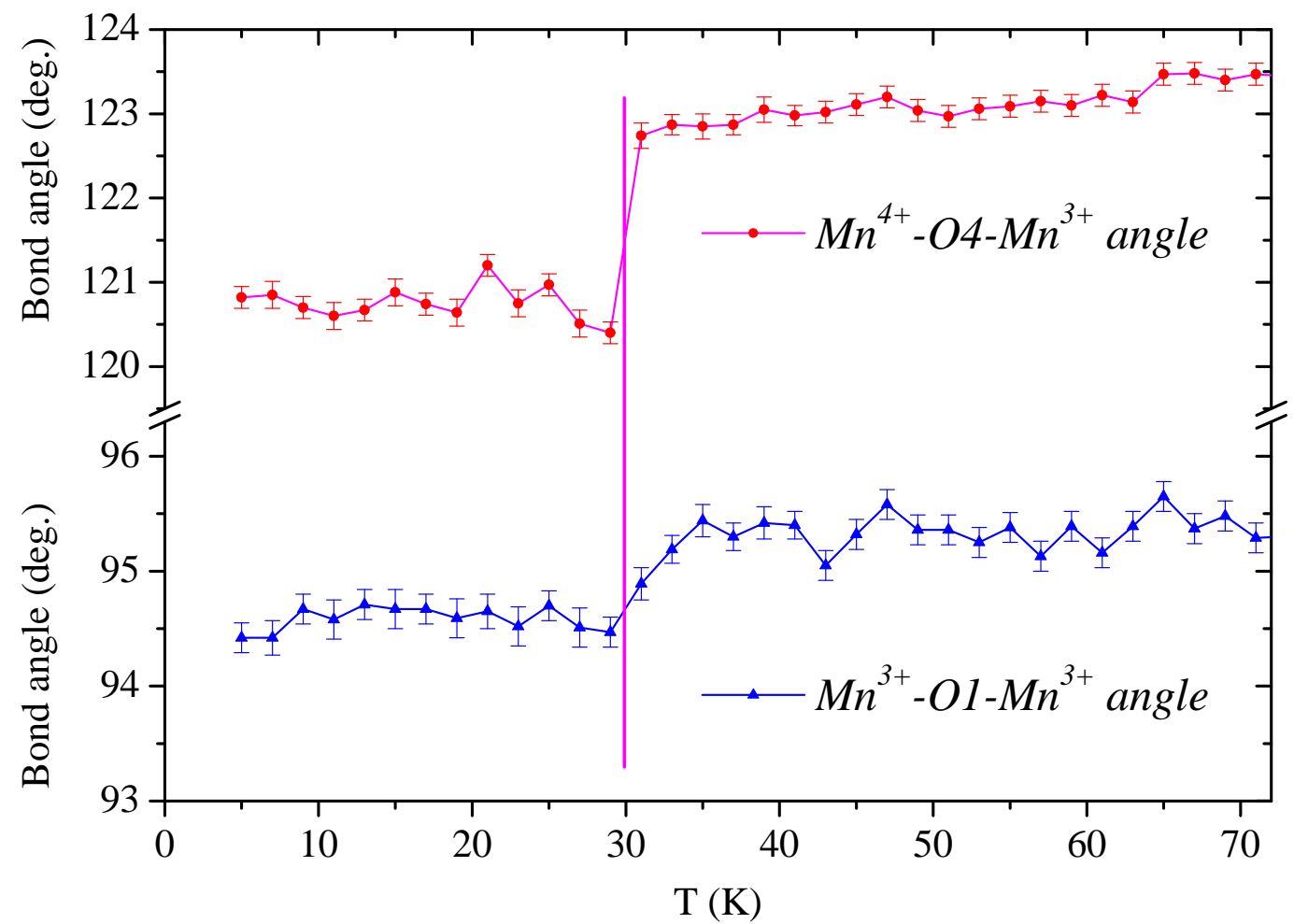

Figure 6: Temperature evolution of the Mn-O-Mn bond angles with temperature. 


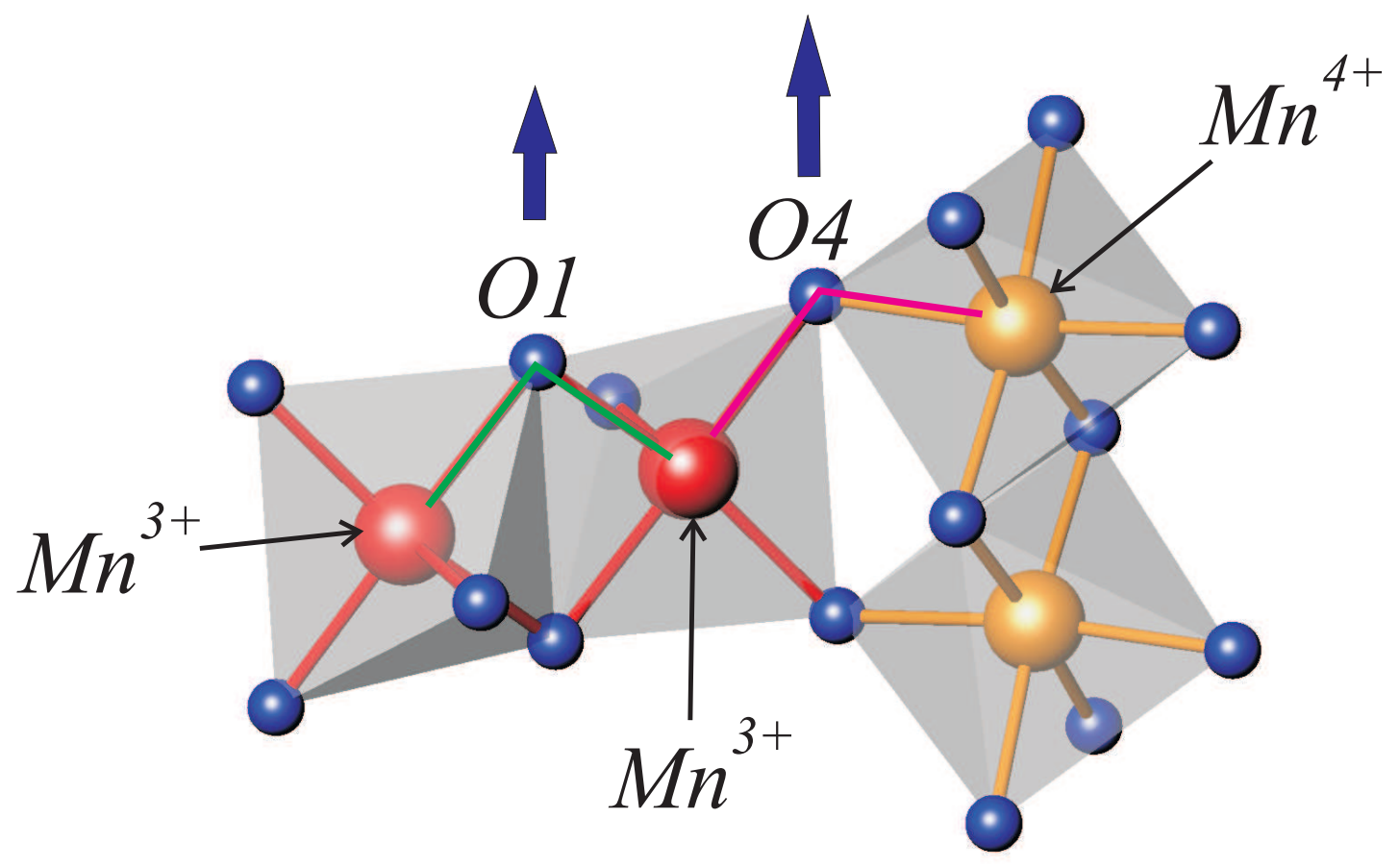

Figure 7: Sketch of the main structural changes in $\mathrm{ErMn}_{2} \mathrm{O}_{5}$. The thick blue arrows indicate the direction of displacements of atoms $\mathrm{O} 1$ and $\mathrm{O} 4$ while cooling below the ferroelectric transition. Positional oxygen parameters at $25 \mathrm{~K}$ : O1: (0, 0, 0.2699), O4: $(0.3945,0.2051,0.2370)$ 